\title{
Impacto prognóstico da expressão imuno-histoquímica do BRCA1 nos carcinomas mamários esporádicos
}

\author{
Prognostic impact of BRCA1 immunohistochemistry expression in sporadic breast carcinomas
}

Alfredo Ribeiro-Silva'; Sérgio Britto Garcia'; Fernando Chahud²;Sérgio Zucoloto

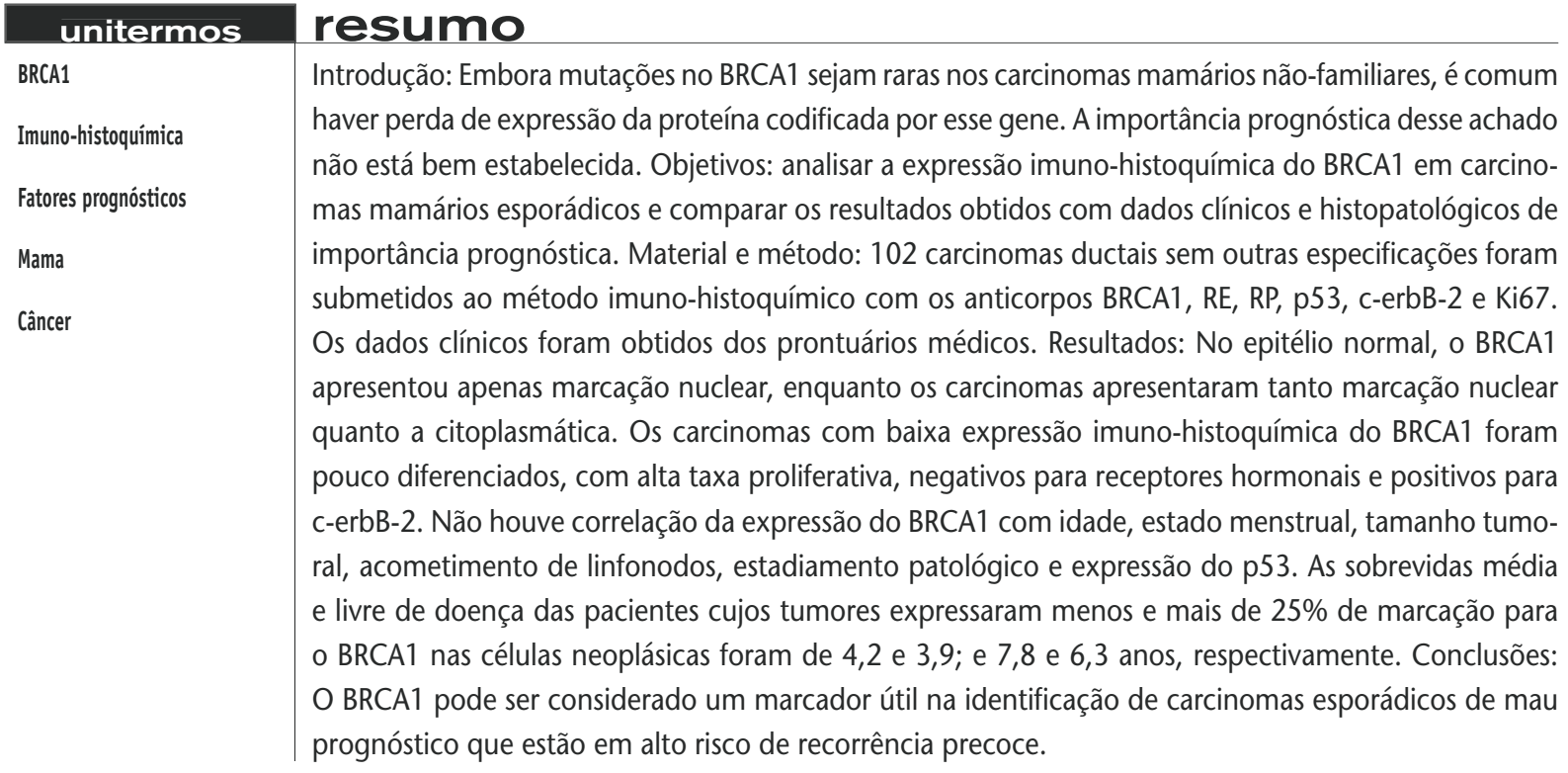

abstract

Background: Although mutations in BRCA are rare in non familiar breast carcinomas, it is common the lost of expression of the protein codified by this gene. The prognostic importance of this finding is not well established. Objectives: to analyze the immunohistochemistry expression of BRCA1 in sporadic breast carcinomas and to compare the results with clinical and histopathologic data of prognostic significance. Material and method: 102 NOS ductal carcinomas were submitted to the immunohistochemistry method with the antibodies BRCA1, ER, $P R, p 53, c-e r b B-2$ and Ki67. The clinical data were retrieved from medical files. Results: In normal epithelium, $B R C A 1$ showed only nuclear staining, while the carcinomas showed either nuclear or cytoplasmatic staining. Carcinomas with reduced expression of BRCA1 were poorly differentiated, with high proliferative rate, negative for hormonal receptors, and positive for c-erbB-2. There was not correlation between BRCA1 expression with age, menstrual status, tumoral size, lymph nodes status, pathologic stage, and p53 expression. The mean survival and disease-free survival of patients which tumors expressed less and more of $25 \%$ of neoplastic cells stained for BRCA1 were 4.2 and 3.9; and 7.8 and 6.3 years respectively. Conclusions: BRCA1 may be considered a useful marker in identification of sporadic carcinomas of poor prognosis that is in high risk for precocious recurrence.

\section{key words} BRCA1

Immunohistochemistry

Prognostic factors

Breast

Cancer

\footnotetext{
1. Professor-doutor do Departamento de Patologia da Faculdade de Medicina de Ribeirão Preto da Universidade de São Paulo (FMRP/USP).

2. Médico-assistente do Serviço de Patologia do Hospital das Clínicas (SERPAT/HC) da FMRP/USP.

3. Professor titular do Departamento de Patologia da FMRP/USP.

Este trabalho contou com o apoio financeiro da Fundação de Amparo à Pesquisa do Estado de São Paulo (FAPESP, processo 03/02532-8).
} 


\section{Introdução}

O gene BRCA1, localizado no cromossomo 17q21, codifica uma proteína nuclear de $220 \mathrm{kDa}$ que participa da regulação do ciclo celular das células epiteliais da glândula mamária. O BRCA1 é considerado um gene supressor tumoral porque mutações nele levam a um defeito no reparo de danos ao DNA e instabilidade genética que favorecem a tumorigênese ${ }^{(3)}$. As mutações hereditárias no BRCA1 estão presentes em cerca de $80 \%$ dos casos de câncer de mama familiar ${ }^{(7)}$. Os pacientes com mutação no BRCA1 apresentam menor tempo de sobrevida livre de doença e maior taxa de mortalidade, assim como maior tendência a bilateralidade ${ }^{(17)}$. Os carcinomas associados a mutações no BRCA1 apresentam perfil fenotípico característico. Tipicamente são carcinomas pouco diferenciados, apresentam elevado grau nuclear e alta taxa proliferativa. Além disso, geralmente são negativos para receptores de estrógeno e c-erbB-2, mas positivos para o p53 ${ }^{(10)}$. Embora mutações no BRCA1 sejam raras nos carcinomas mamários esporádicos, a perda de expressão do RNA mensageiro e da proteína codificada pelo BRCA1 é freqüente nos carcinomas não-familiares, provavelmente devido a fatores epigenéticos ${ }^{(8,18,19)}$. A importância prognóstica desses achados, entretanto, ainda não está bem estabelecida.

\section{Objetivos}

O presente trabalho tem por objetivos analisar a expressão imuno-histoquímica do BRCA1 em carcinomas mamários esporádicos e comparar os resultados obtidos com dados clínicos e histopatológicos de importância prognóstica, incluindo a expressão de receptores hormonais, p53, c-erbB-2 e Ki67.

\section{Material e método}

\section{Casuística}

Esse trabalho segue as normas éticas da Declaração de Helsinque (1972) e foi integralmente aprovado pelo comitê de ética local. Dos arquivos do Serviço de Patologia do Hospital das Clínicas da Faculdade de Medicina de Ribeirão Preto (SERPAT/HCFMRP/USP) foram revisados todos os laudos anatomopatológicos de carcinomas mamários correspondentes aos anos de 1990 a 1992. Foram selecionados para o estudo apenas os carcinomas ductais invasores sem outra especificação, sendo sistematicamente excluídos os carcinomas lobulares e os medulares, assim como as variantes raras do carcinoma ductal. Com base nas lâminas arquivadas coradas por hematoxilina e eosina (HE), os carcinomas ductais foram classificados segundo a graduação de Scarff-Bloom e Richardson modificada por Elston e Ellis(5). Após a análise histológica os carcinomas ductais foram, portanto, divididos em três grupos: grau I (bem diferenciado), grau II (moderadamente diferenciado) e grau III (pouco diferenciado). Nos casos em que a paciente foi submetida a mais de um procedimento diagnóstico, foi considerada apenas a biópsia mais representativa da lesão em estudo. Para o estudo foram consideradas apenas biópsias com material representativo do tumor, ou seja, só foram selecionados os carcinomas que apresentaram tumor em pelo menos dez campos de grande aumento (objetiva de 40x). Além disso, foram excluídas as pacientes submetidas a qualquer esquema de tratamento prévio, como hormonoterapia, quimioterapia e radioterapia. Também foram afastadas todas as pacientes com história de câncer mamário familiar. De acordo com esses critérios, a casuística ficou com 102 casos (34 casos grau I, 39 grau II e 29 grau III). Para controle do trabalho foram utilizadas dez amostras de tecido mamário normal oriundas de pacientes submetidas a mastoplastia redutora durante o mesmo período.

Dos prontuários médicos das pacientes foram obtidos os seguintes dados: idade, estado menstrual, estadiamento patológico, tamanho do nódulo, acometimento linfonodal, presença de metástases, tempo de recorrência e se houve óbito decorrente do carcinoma ou suas complicações. Todas as pacientes são do sexo feminino.

A sobrevida global foi definida como o tempo entre o diagnóstico do carcinoma e o óbito, sendo excluídas dessa análise as pacientes que foram a óbito por motivos não diretamente relacionados ao tumor e as que perderam o seguimento em menos de cinco anos após o diagnóstico. A sobrevida livre de doença foi definida como o tempo entre o diagnóstico e a recorrência ou o aparecimento de metástases. Foram consideradas para essa análise todas as pacientes com seguimento de cinco ou mais anos após o diagnóstico inicial e aquelas que tiveram recorrência ou metástase antes de cinco anos de seguimento.

\section{Método imuno-histoquímico}

A seleção foi feita através da análise de lâminas arquivadas coradas por HE. A partir dos blocos de parafina correspondentes aos fragmentos mais representativos de cada lesão foram feitos cortes histológicos de $3 \mu \mathrm{m}$, que foram colocados em lâminas de vidro. Esses fragmentos foram 
submetidos à técnica imuno-histoquímica pelo método de avidina-biotina-peroxidase, utilizando-se o kit universal da Novocastra ${ }^{\circledR}$ (Novostain Super ABC Kit Universal), segundo protocolo desenvolvido pelo Laboratório de Proliferação celular do Departamento de Patologia da FMRP/USP(12). Foram utilizados os seguintes anticorpos primários, nas seguintes diluições: BRCA1 (1:100, clone MS13, Serotec ${ }^{\circledR}$, Oxford, UK), receptor de estrógeno (1:100, clone 6F11, Novocastra $^{\circledR}$, Newcastle Upon Tyne, UK), receptor de progesterona $\left(1: 150\right.$, clone 16 , Novocastra ${ }^{\circledR}$, Newcastle Upon Tyne, UK), p53 (1:100, clone DO-7, Novocastra ${ }^{\circledR}$, Newcastle Upon Tyne, UK), c-erbB-2 (1:200, clone CB11, Novocastra ${ }^{\circledR}$, Newcastle Upon Tyne, UK ) e Ki67 (1:150, clone MM1, Novocastra ${ }^{\circledR}$, Newcastle Upon Tyne, UK). Para revelação das reações foi utilizada a diaminobenzidina (DAB) $\left(\right.$ Sigma $\left.^{\circledR}\right)$. Após a reação, as lâminas foram cobertas por lamínula, com auxílio de Permount (Fischer $\left.{ }^{\circledR}\right)$.

Todos os marcadores foram utilizados com controles externos positivos e negativos. Para BRCA1 e receptores hormonais os controles positivos foram amostras de tecido mamário normal. Para p53, c-erbB-2 e Ki67 foram utilizados como controle carcinomas mamários sabidamente positivos para esses marcadores. O controle negativo foi feito pela omissão do anticorpo primário.

As lâminas foram analisadas em microscópio de luz convencional, sendo consideradas células positivas aquelas coradas de marrom escuro após as reações. Para a interpretação do BRCA1 foi considerada marcação tanto nuclear quanto citoplasmática. Os carcinomas foram graduados da seguinte maneira: 0/+ (negatividade ou positividade em menos de $25 \%$ das células neoplásicas), ++ (positividade entre $25 \%$ e $50 \%$ das células neoplásicas) e +++ (positividade em mais de $50 \%$ das células neoplásicas) ${ }^{(9)}$. Para a análise de receptores hormonais e $\mathrm{p} 53$ foram considerados positivos os casos com $10 \%$ ou mais de positividade nuclear nas células neoplásicas para esses marcadores ${ }^{(4,14)}$. A análise do c-erbB-2 e do Ki67 foi feita conforme recomendações do Colégio Americano de Patologistas(5).

\section{Análise estatística}

Os resultados foram submetidos a análise estatística utilizando o software Graph Pad Prism 4.0 (San Diego, USA). Para verificar a correlação entre os parâmetros avaliados foram utilizados os testes exato de Fisher, para dois grupos de dados, e qui-quadrado, quando havia três ou mais grupos a serem analisados. As curvas de sobrevida foram feitas de acordo com o método de Kaplan-Meier, e a comparação entre as curvas, pelo teste log-rank. As diferenças estatísticas foram consideradas significantes para $\alpha=5 \%$.

\section{Resultados}

No epitélio ductal e lobular normal, o BRCA1 apresentou apenas marcação nuclear, enquanto os carcinomas apresentaram tanto marcação nuclear quanto a citoplasmática (Figura 1).

A expressão imuno-histoquímica do BRCA1 correlacionou-se com o grau tumoral $(p<0,001)$. De acordo com a Tabela 1, observa-se que os carcinomas pouco diferenciados (grau III) tiveram menor expressão do BRCA1 do que os bem diferenciados (grau I). Não houve correlação estatisticamente significante da expressão do BRCA1 com idade, estado menstrual, tamanho tumoral, acometimento de linfonodos e estadiamento patológico.



Figura 1 - Expressão imuno-histoquímica do BRCA1 em carcinomas mamários. A: marcação nuclear e citoplasmática (400x); B: marcação predominantemente citoplasmática (400x) 
Tabela 1 Expressão do BRCA1 em relação aos parâmetros clinicopatológicos avaliados

\begin{tabular}{|c|c|c|c|c|}
\hline Característica & $\begin{array}{c}\text { BRCA1 -/+ } \\
\text { n/\% }\end{array}$ & $\begin{array}{c}\text { BRCA1 ++ } \\
\text { n/\% }\end{array}$ & $\begin{array}{c}\text { BRCA1 +++ } \\
\text { n/\% }\end{array}$ & $p$ \\
\hline Total & $26(25,5)$ & $26(25,5)$ & $50(49)$ & \\
\hline \multicolumn{5}{|l|}{ Idade (anos) } \\
\hline$<30$ & $1(0,9)$ & $1(0,9)$ & $1(0,9)$ & 0,5857 \\
\hline $30-50$ & $8(7,8)$ & $6(5,8)$ & $17(16,6)$ & \\
\hline $50-70$ & $9(8,8)$ & $15(14,7)$ & $24(23,5)$ & \\
\hline$>70$ & $8(7,8)$ & $4(3,9)$ & $8(7,8)$ & \\
\hline \multicolumn{5}{|l|}{ Estado menstrual } \\
\hline Pré-menopausa & $9(8,8)$ & $8(7,8)$ & $17(16,6)$ & 0,9546 \\
\hline Pós-menopausa & $17(16,6)$ & $17(16,6)$ & $31(30,4)$ & \\
\hline Histerectomizada & 0 & $1(0,9)$ & $2(1,9)$ & \\
\hline \multicolumn{5}{|c|}{ Tamanho tumoral (cm) } \\
\hline$<2$ & $1(0,9)$ & $6(5,8)$ & $10(9,8)$ & 0,1706 \\
\hline $2-5$ & $15(14,7)$ & $12(11,7)$ & $30(29,4)$ & \\
\hline$>5$ & 10 & 8 & 10 & \\
\hline \multicolumn{5}{|c|}{ Grau tumoral (B \& R) } \\
\hline I & $2(1,9)$ & $4(3,9)$ & $28(27,4)$ & $<0,0001$ \\
\hline II & $1(0,9)$ & $17(16,6)$ & $21(20,6)$ & \\
\hline III & $23(22,5)$ & $5(4,9)$ & $1(0,9)$ & \\
\hline \multicolumn{5}{|c|}{ Linfonodos axilares } \\
\hline Negativo & $7(6,8)$ & $10(9,8)$ & $16(15,7)$ & 0,8266 \\
\hline $1-3$ & $7(6,8)$ & $6(5,8)$ & $16(15,7)$ & \\
\hline$>3$ & $12(11,7)$ & $10(9,8)$ & $18(17,6)$ & \\
\hline \multicolumn{5}{|c|}{ Estadiamento patológico } \\
\hline I & 0 & $2(1,9)$ & $6(5,8)$ & 0,307 \\
\hline IIa & $8(7,8)$ & $6(5,8)$ & $11(10,8)$ & \\
\hline IIb & $3(2,9)$ & $5(4,9)$ & $12(11,7)$ & \\
\hline IIIa & $7(6,8)$ & $5(4,9)$ & $9(8,8)$ & \\
\hline IIIb & 0 & $1(0,9)$ & 0 & \\
\hline IIIC & $7(6,8)$ & $7(6,8)$ & $7(6,8)$ & \\
\hline IV & $1(0,9)$ & 0 & $5(4,9)$ & \\
\hline
\end{tabular}

BRCA1 -/+: negatividade ou positividade em menos de 25\% das células neoplásicas; BRCA1 ++: positividade entre 25\% e $50 \%$ das células neoplásicas; BRCA1 +++: positividade em mais de 50\% das células neoplásicas.

Segundo a Tabela 2, a expressão reduzida do BRCA1 correlacionou-se com negatividade para os receptores de estrógeno e progesterona e positividade para o c-erbB-2. Carcinomas com baixa expressão do BRCA1 também apresentaram alta taxa proliferativa, conforme resultados obtidos pelo Ki67 (Tabela 2). Não houve associação estatística entre a expressão do BRCA1 e a do p53.

Das 102 pacientes incluídas no trabalho, 30 foram a óbito em decorrência do tumor. Trinta e três permaneceram vivas após cinco anos do diagnóstico, sendo que 25 delas tiveram sobrevida igual ou superior a dez anos após o diagnóstico inicial. Trinta e nove pacientes foram excluídas dessa análise porque não tiveram seguimento de cinco anos ou porque faleceram por motivos não-relacionados ao carcinoma. A sobrevida média das pacientes cujos tumores expressaram menos de $25 \%$ de marcação para o BRCA1 nas células neoplásicas foi de 4,2 anos. As pacientes com expressão do BRCA1 em mais de $25 \%$ das células neoplásicas tiveram sobrevida média de 7,8 anos. Essa diferença foi estatisticamente significativa $(p<0,001)$ (Figura 2). 


\section{Tabela 2 Expressão do BRCA1 em relação aos marcadores imuno-histoquímicos avaliados}

\begin{tabular}{|c|c|c|c|c|}
\hline Característica & $\begin{array}{c}\text { BRCA1 -/+ } \\
\text { n/\% }\end{array}$ & $\begin{array}{c}\text { BRCA1 ++ } \\
\text { n/\% }\end{array}$ & $\begin{array}{c}\text { BRCA1 +++ } \\
\text { n/\% }\end{array}$ & $p$ \\
\hline Total & $26(25,5)$ & $26(25,5)$ & $50(49)$ & \\
\hline \multicolumn{5}{|l|}{ RE } \\
\hline Positivo & $7(6,8)$ & $12(11,7)$ & $35(34,3)$ & 0,0012 \\
\hline Negativo & $19(18,6)$ & $14(13,7)$ & $15(14,7)$ & \\
\hline \multicolumn{5}{|l|}{ RP } \\
\hline Positivo & $6(5,8)$ & $12(11,7)$ & $30(29,4)$ & 0,0092 \\
\hline Negativo & $20(19,6)$ & $14(13,7)$ & $20(19,6)$ & \\
\hline \multicolumn{5}{|l|}{ P53 } \\
\hline Positivo & $8(7,8)$ & $13(12,7)$ & $14(13,7)$ & 0,1446 \\
\hline \multicolumn{5}{|l|}{ c-erbB-2 } \\
\hline 0 & $12(11,7)$ & $11(10,8)$ & $35(34,3)$ & 0,0312 \\
\hline $1+$ & $3(2,9)$ & $6(5,8)$ & $8(7,8)$ & \\
\hline $2+$ & $5(4,9)$ & $3(2,9)$ & 0 & \\
\hline $3+$ & $6(5,8)$ & $6(5,8)$ & $7(6,8)$ & \\
\hline \multicolumn{5}{|l|}{ Ki67 } \\
\hline$<10 \%$ & $8(7,8)$ & $13(12,7)$ & $34(33,3)$ & 0,026 \\
\hline $11-50 \%$ & $11(10,8)$ & $7(6,8)$ & $12(11,7)$ & \\
\hline$>51 \%$ & $7(6,8)$ & $6(5,8)$ & $4(3,9)$ & \\
\hline
\end{tabular}

BRCA1 -/+: negatividade ou positividade em menos de 25\% das células neoplásicas; BRCA1 ++: positividade entre 25\% e $50 \%$ das células neoplásicas; BRCA1 +++: positividade em mais de $50 \%$ das células neoplásicas.

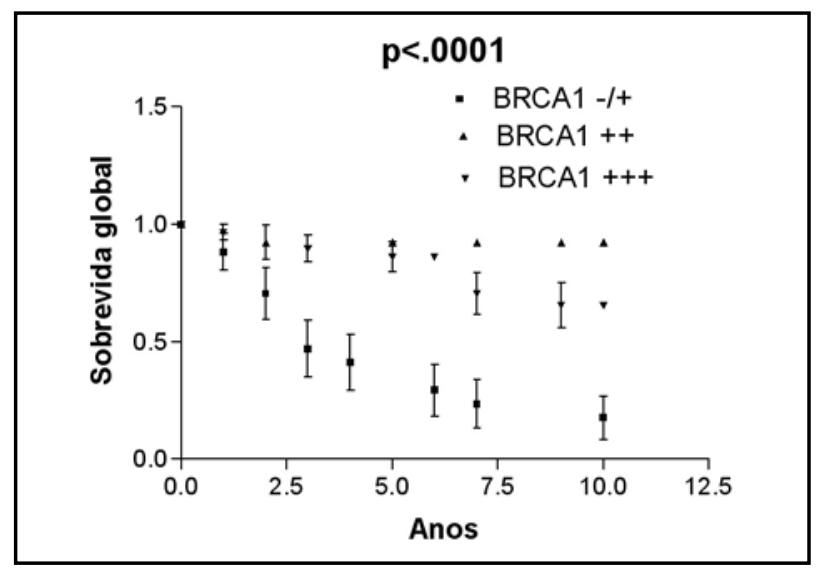

Figura 2 - Taxa de sobrevida global em relação à reportagem de expressão do BRCA1

Das 102 pacientes incluídas no trabalho, 46 apresentaram recorrência ou metástase. Vinte e seis permaneceram livres da doença cinco anos após o tratamento, sendo que 19 delas não tiveram recorrência nem metástases após dez anos do diagnóstico inicial. Trinta pacientes foram excluídas dessa análise porque não tiveram seguimento de cinco anos. A sobrevida livre de doença média das pacientes cujos tumores expressaram menos de $25 \%$ de marcação para o BRCA1 nas células neoplásicas foi de 3,9 anos. As pacientes com expressão do BRCA1 em mais de $25 \%$ das células neoplásicas tiveram sobrevida livre de doença média de 6,3 anos. Essa diferença foi estatisticamente significativa $(p=0,0095)$ (Figura 3).

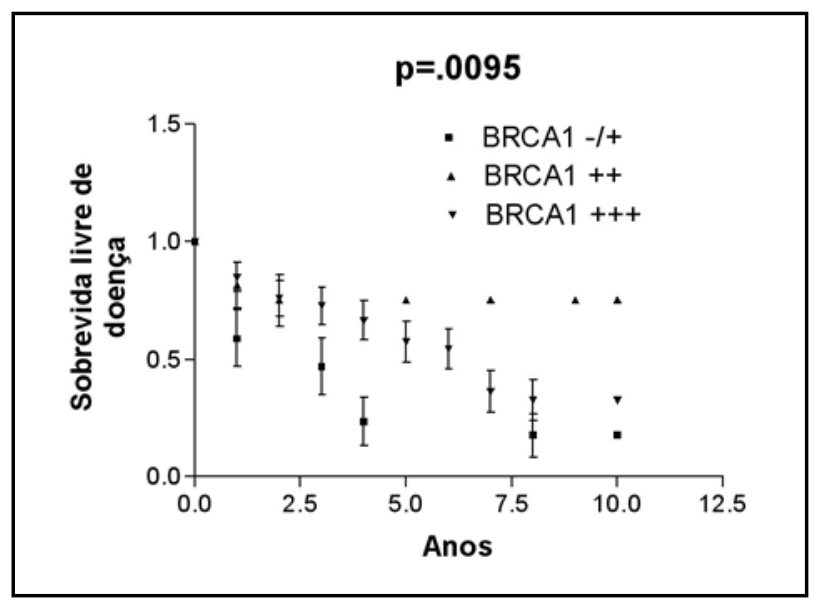

Figura 3 - Taxa de sobrevida livre de doença em relação à porcetagem de expressão do BRCA1 


\section{Discussão}

Mutações hereditárias no gene BRCA1 estão associadas ao câncer de mama familiar, que representa 5\% a $10 \%$ de todos os carcinomas mamários. Entretanto, a grande maioria dos carcinomas mamários é de ocorrência esporádica, ou seja, não-familiar, e, mesmo nesses casos, pode-se observar diminuição dos níveis de RNA mensageiro associada a menor expressão imuno-histoquímica do $\mathrm{BRCA}^{(6,7)}$. A baixa expressão imuno-histoquímica verificada nesses casos não está associada a mutações no gene, e sim a fatores epigenéticos, como metilação no DNA, perda da heterozigosidade e defeito no transporte intracelular do produto do gene BRCA1 ${ }^{(11)}$.

O padrão de marcação imuno-histoquímica do BRCA1 é diferente nos carcinomas mamários em relação ao tecido mamário normal. Enquanto a marcação no epitélio normal é exclusivamente nuclear, a nos carcinomas pode ser exclusivamente nuclear, predominantemente nuclear, predominantemente citoplasmática ou exclusivamente citoplasmática ${ }^{(1,18)}$. Na célula normal a proteína codificada pelo BRCA1 é sintetizada no citoplasma e transportada até o núcleo, onde desempenha seu papel regulador do ciclo celular. Acredita-se que no carcinoma ocorra um defeito nesse transporte, justificando sua expressão citoplasmática( ${ }^{(7)}$.

Os carcinomas que apresentam expressão reduzida do BRCA1 tipicamente são pouco diferenciados(2,16,18). A baixa expressão do BRCA1 é encontrada em todas as variantes histológicas do carcinoma mamário, incluindo os carcinomas lobulares, medulares, mucinosos e intraductais ${ }^{(8)}$. O BRCA1 correlaciona-se inversamente com o grau histológico, mas não há relação com o acometimento de linfonodos ou outros parâmetros prognósticos ${ }^{(19)}$.

Em nossa casuística, 51\% dos carcinomas apresentaram baixa expressão do BRCA1, o que está ligeiramente acima do esperado segundo a literatura (cerca de $40 \%)^{(16)}$. De acordo com os nossos resultados, os carcinomas com expressão reduzida do BRCA1 são de alto grau histológico e apresentam alta taxa proliferativa, confirmada pela expressão do Ki67. São carcinomas negativos para os receptores de estrógeno e os de progesterona, mas positivos para o c-erbB-2. Não houve correlação estatisticamente significativa entre expressão imuno-histoquímica do BRCA1 e idade, estado menstrual, tamanho tumoral, acometimento de linfonodos e estadiamento patológico. Ao contrário do esperado, segundo a literatura, não houve correlação entre a expressão do BRCA1 e o p53 ${ }^{(10)}$.

Não existe um consenso acerca do papel prognóstico da expressão imunoistoquímica do BRCA1 em carcinomas mamários, e os dados disponíveis são discrepantes entre si. Alguns autores afirmam que a expressão reduzida do BRCA1 está associada a pior prognóstico, enquanto outros alegam que o comportamento biológico dos carcinomas mamários não é alterado pela porcentagem de células tumorais positivas para esse marcador ${ }^{(3,13,19)}$. Acirrando essa polêmica, Lambie et al. verificaram que, entre carcinomas de mesmo grau histológico, aqueles que apresentam perda da expressão do BRCA1 possuem maior tempo de sobrevida livre de doença, sugerindo que o BRCA1 não seja um fator prognóstico independente ${ }^{(6)}$. Esses resultados, entretanto, foram refutados por Dorssers et al. ${ }^{(3)}$.

De acordo com nossos resultados, os carcinomas com baixa expressão imuno-histoquímica do BRCA1 apresentam sobrevida global e tempo livre de doença (recorrência ou metástases) inferiores aos dos carcinomas com elevada expressão desse marcador. Esses dados justificam o uso do BRCA1 em painéis de imuno-histoquímica, visto que ele pode ser utilizado como um indicador de mau prognóstico. Além disso, a baixa expressão do BRCA1 está associada a metástases à distância nos carcinomas mamários não-familiares, sugerindo que o BRCA1 possa exercer algum papel na progressão tumoral dos carcinomas esporádicos ${ }^{(15,20)}$.

\section{Conclusão}

O BRCA1 pode ser considerado um marcador útil na identificação de mulheres com carcinoma esporádico de mau prognóstico que estão em alto risco de recorrência precoce. A baixa imunoexpressão da proteína do BRCA1 em carcinomas mamários esporádicos e sua correlação com fatores de mau prognóstico indicam que o grau de expressão desse gene pode desempenhar importante papel na patogênese do carcinoma mamário não-familiar.

\section{Agradecimentos}

Os autores agradecem o apoio técnico de Deisy Mara da Silva, Auristela de Mello Martins, Laura Midori Kawasse e Márcia Aparecida Ferreira Oliva.

\section{Referências}

I. CHAMBON, M. et al. Localization of BRCAI protein in human breast cancer cells. Breast Cancer Res Treat, v. 79, n. I, p. 107-19, 2003.
2. DING, S. L. et al. Abnormality of the DNA double-strand-break checkpoint/repair genes, ATM, BRCAI and TP53, in breast cancer is related to tumour grade. $\mathrm{Br}$ J Cancer, v. $90, \mathrm{n} .10$, 
p. 1995-200I, 2004.

3. DORSSERS, L. C. et al.The prognostic value of BRCAI in patients with primary breast cancer. Clin Cancer Res, v. 10, n. I8, p. 6194-202, 2004.

4. FERRERO-POÜS, M. et al. Comparison of enzyme immunoassay and immunohistochemical measurements of estrogen and progesterone receptors in breast cancer patients. Appl Immunohistochem, v. 9, p. 267-75, 2001.

5. FITZGIBBONS, P. L. et al. Prognostic factors in breast cancer: College of American Pathologists Consensus Statement 1999. Arch Pathol Lab Med, v. 124, p. 966-78, 2000.

6. LAMBIE, H. et al. Prognostic significance of BRCAI expression in sporadic breast carcinomas. J Pathol, v. 200, n. 2, p. 20713,2003.

7. LEE,W.Y. et al. Immunolocalization of BRCAI protein in normal breast tissue and sporadic invasive ductal carcinomas: a correlation with other biological parameters. Histopathology, v. 34, n. 2, p. 106-12, 1999.

8. MAGDINIER, F. et al. Down-regulation of BRCAI in human sporadic breast cancer; analysis of DNA methylation patterns of the putative promoter region. Oncogene, v. 17 , n. 24, p. 3169-76, 1998.

9. NIETO,A. et al. BRCA I expression in canine mammary dysplasias and tumours: relationship with prognostic variables.J Comp Pathol, v. 128, n. 4, p. 260-8, 2003.

I0. OSIN, P.P.; LAKHANI S. R. The pathology of familial breast cancer: immunohistochemistry and molecular analysis. Breast Cancer Res, v. I, n. I, p. 36-40, 1999.

II. PEREZ-VALLES, A. et al. The usefulness of antibodies to the BRCAI protein in detecting the mutated BRCAI gene.
An immunohistochemical study. J Clin Pathol, v. 54, n. 6, p. 476-80, 200 I.

12. RIBEIRO-SILVA, A. et al.The relationship between p63 and p53 expression in normal and neoplastic breast tissue. Arch Pathol Lab Med, v. I27, n. 3, p. 336-40, 2003.

13. ROSEN, P. P. Rosen's Breast Pathology. 2 ed. Philadelphia, P. A.: Lippincott Williams \& Wilkins; 2001 .

14. RUDOLPH, P. et al. Concurrent overexpression of p53 and cerbB-2 correlates with accelerated cycling and concomitant poor prognosis in node-negative breast cancer. Hum Pathol, v. 32, p. 3 I I-9, 200 I.

15. SEERY, L. T. et al. BRCAI expression levels predict distant metastasis of sporadic breast cancers. Int J Cancer, v. 84, n. 3, p. 258-62, 1999.

16.TAYLOR, J. et al. An important role for BRCAI in breast cancer progression is indicated by its loss in a large proportion of nonfamilial breast cancers. Int J Cancer, v. 79, n. 4, p. 334-42, 1998.

17. VERHOOG, L. C. et al. Survival and tumour characteristics of breast-cancer patients with germline mutations of BRCAI. Lancet, v. 351, n. 9099, p. 316-21, 1998.

I8.WILSON, C. A. et al. Localization of human BRCAI and its loss in high-grade, non-inherited breast carcinomas. Nat Genet, v. 21, n. 2, p. 236-40, 1999.

19. YANG, Q. et al. Prognostic significance of BRCAI expression in Japanese sporadic breast carcinomas. Cancer, v. 92, n. I, p. 54-60, 2001.

20.YOSHIKAWA, K. et al. Reduction of BRCAI protein expression in Japanese sporadic breast carcinomas and its frequent loss in BRCAI-associated cases. Clin Cancer Res, v. 5, n. 6, p. |249-61, 1999.

\begin{tabular}{l|l} 
& Endereço para correspondência \\
\hline & Alfredo Ribeiro-Silva \\
Departamento de Patologia - Laboratório de \\
Patologia Cinecológica e Mamária \\
Faculdade de Medicina de Ribeirão Preto da \\
Universidade de São Paulo \\
Av. Bandeirantes, 3.900 - Campus Universitário \\
Monte Alegre \\
CEP 14049-900 - Ribeirão Preto-SP \\
Tels.: (16) 602-3119/602-3244 \\
Fax: (16) 633-0095/633-1068 \\
e-mail: arsilva@fmrp.usp.br
\end{tabular}

